\title{
Odontoma composto como fator de impactação dentária: Relato de caso
}

\author{
Odontoma composed as a tooth impact factor: Case report
}

\author{
Odontoma compuesto como factor de impacto dental: Reporte de un caso
}

Arthur Eric Costa Wanderley ${ }^{1 *}$, Rúbia Reis Fonseca Amaral Souto ${ }^{1}$, Mayssa Galvão Pimentel ${ }^{1}$, Ingrid Ferreira Leite ${ }^{1}$, Ellen Souza Marques ${ }^{1}$, Sofia Virna Jucá Dantas Melo ${ }^{1}$, Áurea Valéria de Melo Franco ${ }^{1}$, Lucas Fortes Cavalcanti de Macêdo'

\begin{abstract}
RESUMO
Objetivo: Descrever um caso clínico de odontoma composto, avaliando seus sinais e sintomas mais comuns, a sua localização, idade e gênero do paciente, as alterações histopatológicas e seu tratamento mais adequado, enfatizando a relevância da radiografia na prática odontológica. Relato de caso: Numa investigação de dente não erupcionado, pode-se observar um aumento de volume devido à tentativa de erupção do dente 13. Com isso, planejou-se a exérese desta lesão. Quanto ao pós-operatório, o paciente evoluiu sem alterações funcionais ou sensoriais, e após 7 dias, foi verificado um ótimo início de cicatrização tecidual. Paciente encontra-se no aguardo do planejamento do tracionamento ortodôntico do dente impactado. Considerações finais: Ressalta-se a importância da investigação radiográfica em casos dentes não erupcionados, erupção retardada ou retenção de dentes decíduos ou permanentes, pois pode favorecer o diagnóstico precoce. Preconiza-se a remoção cirúrgica por meio dos princípios de exodontia de elementos dentários inclusos como tratamento, sua recidiva é rara.
\end{abstract}

Palavras-chave: Radiografia Panorâmica, Odontoma, Dente não Erupcionado.

\begin{abstract}
Objective: The aim os the present study is report one clinical case of compound ondontoma, analyzing the most commom signals ans symptoms, its location, affected ages and genre of the patient, histopathoogicals changes and the appropriate treatment, enphasizing the radiological tests in a clinical routine. Case report: Investigation of a permanent tooth unerupted, removal of the lesion was planned. The postoperative evolve unchanged functional or sensory after 7 days. Patient is waiting for planned tooth orthodontic treatment. Conclusion: The importance of an radiographic investigation in unerupted tooth, delayed eruption or deciduous or permanente teeth eruption retention can help to a previous diagnose. The treatment by surgery tooth removal, its relapse is rare.
\end{abstract}

Key Words: Panoramic Radiography, Odontoma, Tooth Unerupted.

\section{RESUMEN}

Objetivo: Describir un caso clínico de odontoma compuesto, evaluando sus signos y síntomas más comunes, su ubicación, edad y sexo, cambios histopatológicos y su tratamiento más apropiado, enfatizando la relevancia de la radiografía en la práctica clinica. Métodos: Descripción del presente caso. Informe del caso: Investigación de un diente permanente retenido, se planificó la extirpación de la lesión. En cuanto al período postoperatorio, evolucionó sin alteraciones funcionales o sensoriales, después de 7 días, un excelente inicio de curación del tejido. El paciente está esperando la planificación de la tracción ortodóncica del diente impactado. Conclusión: Enfatizamos la importancia de la investigación radiográfica en casos de dientes no erupcionados, erupción tardía o retención de dientes deciduos o permanentes, ya que puede favorecer el diagnóstico precoz. La extracción quirúrgica es recomendada por los principios de extracción de elementos dentales incluidos como tratamiento, su recurrencia es rara.

Palabras-Clabe: Radiografía Panorámica, Odontoma, Diente retenido.

\footnotetext{
${ }^{1}$ Centro Universitário Cesmac (CESMAC), Maceió-Alagoas. *E-mail: arthur.ecw@gmail.com
} 


\section{INTRODUÇÃO}

Os tumores odontogênicos são lesões heterogêneas derivadas dos tecidos epiteliais ou ectomesenquimais. Vão desde proliferações não neoplásicas até neoplasias malignas com capacidade metastática. Nos seres humanos os tumores odontogênicos são comparativamente raros, compreendendo cerca de $1 \%$ de todas as amostras de biópsia oral e maxilo-facial diagnosticadas (AHIRE MS, et al., 2018). Estudos relatam que dentre os principais tumores odontogênicos a lesão mais comumente diagnosticada é o odontoma, seguido de ameloblastoma, tumor odontogênico adenomatóide e mixoma odontogênico (SANTOS JN, et al., 2001),

A Organização Mundial da Saúde (OMS) classificou os odontomas como tumores odontogênicos benignos mistos, segundo Pires WR, et al. (2013), formado por estruturas dentárias, isto é, esmalte e dentina e em alguns casos, cemento e polpa em diferentes graus de desenvolvimento (DE OLIVEIRA BH, et al., 2001). Geralmente, são considerados como malformações de desenvolvimento dos tecidos dentais em vez de neoplasias verdadeiras, já que tanto os componentes epiteliais quanto os ectomesenquimais têm células morfologicamente normais com arranjo estrutural defeituoso (GONZALEZ-ALVA P, et al., 2011). Correspondem de $20 \%$ a $67 \%$ dos casos, sem predileção por gênero, conforme Pires WR, et al. (2013), e sendo diagnosticados principalmente na segunda década de vida. São lesões assintomáticas e conhecidos como "achados radiográficos", geralmente associados a investigação de dentes inclusos, aumento de volume local e/ou processos infecciosos (KURAMOCHI MM, et al., 2006).

A OMS em 2005 classificou os odontomas do ponto de vista histopatológico, como: complexos, de acordo com Akerzoul N, et al. (2017), onde os tecidos dentários são bem formados, mas exibem um arranjo mais ou menos desarranjado compondo-se de dentina primária ou imatura como parte principal; é circundado por tecido conjuntivo fibroso e possui preferência pela região posterior da mandíbula, em concordância com Pires WR, et al. (2013), e odontomas compostos, que transcorre da repartimento da papila dentária normal em duas ou mais, promovendo estruturas dentárias normais, mas seu tamanho e sua conformação são alterados, originando múltiplos dentes pequenos semelhantes a estruturas chamadas dentículos. Em geral, os tumores compostos são mais frequentes do que os complexos (KODALI RM, et al., 2010; BARNES L, et al., 2005). Onde aqueles ocorrem mais frequentemente nas regiões molares posteriores, e estes tendem a se desenvolver na região anterior da maxila (ABRAHAMS JM e MCCLURE SA, 2016).

A etiologia da lesão é desconhecida, mas acredita-se na associação de alguns fatores como trauma em dentes decíduos, processo inflamatório, anomalias hereditárias e mutações genéticas, em conformidade com Kämmerer PW, et al. (2016) e Pippi R (2014), distúrbios de erupção, incluindo impactação, erupção retardada ou retenção de dentes decíduos ou permanentes (ISOLA G, et al., 2017). Geralmente são indolores e não agressivos, onde após a excisão, apresentam raríssimos casos recidivantes, exceto em casos onde a lesão foi removida nos estágios iniciais de sua formação (PREOTEASA CT e PREOTEASA E, 2018).

Radiograficamente, os odontomas demonstram radiopacidade bem delimitada, com densidade mais elevada que a do tecido ósseo próximo e similar à dentária. No odontoma composto, analisam-se inúmeras estruturas semelhantes a dentes com medidas diversificadas e desordenadas envoltas por área radiolúcida, onde essas particularidades para autores como Henriksson CO e Kjellman O (1964) e Freitas A, et al. (1998) é apontada como imagem radiográfica patognomônica do odontoma composto. O odontoma complexo, todavia, apresenta-se como uma massa radiopaca irregular demarcada por área radiolúcida possibilitando a necessidade de formação do diagnóstico diferencial.

De acordo com o diagnóstico diferencial de lesões situadas entre raízes dentárias, considera-se a osteíte focal residual, cementoma, tumor odontogênico epitelial calcificante, tumor odontogênico adenomatóide, dentes supranumerários, fibroma cementificante ou osteoblastoma benigno. Caso a lesão esteja situada na região pericoronária, o diagnóstico diferencial engloba tumor odontogênico adenomatóide, tumor odontogênico epitelial calcificante, fibrodentinoma ameloblástico ou odontoameloblastoma. Os tumores ósseos que podem se aproximar radiograficamente com o odontoma complexo são osteoma osteóide, osteoblastoma, cementoblastoma e fibroma cemento-ossificante (CHRCANOVIC RB, et al., 2010). 
O tratamento proposto para o odontoma composto, assim como para o complexo, é a remoção cirúrgica através dos fundamentos de exodontia de elementos dentários inclusos, conforme Cardoso LC et al. (2003), e por serem desmembrados do osso por uma película de tecido conjuntivo fibroso, essas lesões normalmente são de fácil remoção (CHRCANOVIC RB, et al., 2010). Estudos evidenciam a incidência de odontomas relacionados a elementos dentários, do qual o tratamento abrange a remoção do tumor com ou sem dente impactado, cuja erupção pode sobrevir de maneira espontânea ou, através de tracionamento ortodôntico que ocorre de forma mais frequente (NASCIMENTO MM, et al., 2016).

Apesar de os odontomas serem lesões de especificidade benigna e possam ser facilmente diagnosticados, os profissionais da odontologia devem estar bem habituados com a manifestação clínica, assim como com a apresentação radiológica da mesma. Ocasionalmente, a lesão alcança um tamanho grande infrequente podendo evidenciar assimetria facial (AKERZOUL N, et al., 2017).

Este trabalho objetiva descrever e relatar as características clínicas, radiográficas e histopatológicas do odontoma composto, enfatizando a relevância da radiografia diagnóstica na prática odontológica afim de localizar lesões quer sejam benignas, quer sejam malignas, mas que ainda não foram diagnosticadas clinicamente.

\section{RELATO DE CASO}

Paciente do gênero masculino, 35 anos, procurou atendimento odontológico alegando "minha presa não nasceu e fui a um dentista que disse que eu tinha uns 30 dentes a mais do que deveria".

Durante a anamnese informou ser fumante e etilista, não possuir nenhuma alteração sistêmica e que há 1 mês realizou exame radiográfico para investigação de ausência do dente 13, porém ainda sem resultado.

Durante a avaliação intrabucal, observou-se ausência do dente 13, discreto aumento de volume e possível tentativa de irrupção de estrutura compatível com esmalte dentário (Figura 1).

Figura 1 - Aspecto clínico inicial.

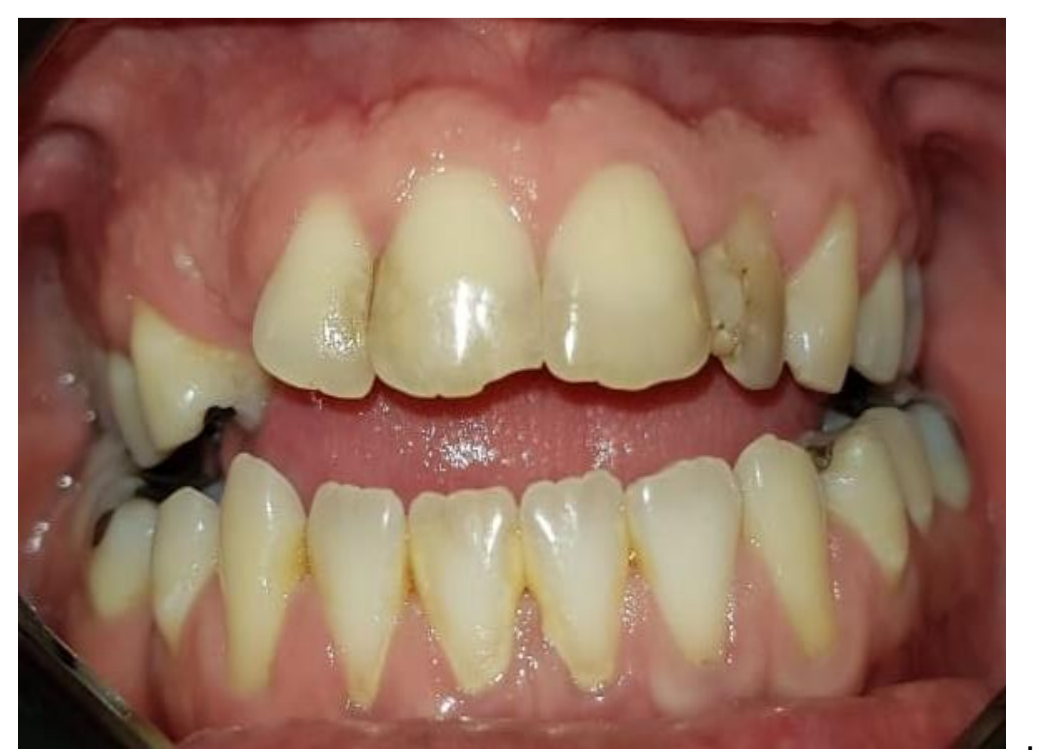

Fonte: Wanderley AEC, et al., 2019.

Foi solicitado uma nova radiografia panorâmica a qual demonstrou presença de radiopacidade compatível com tecidos calcificados em região anterior de maxila associado a impactação do dente 13, (Figura 2). Diante das características clínicas e radiográficas, a hipótese diagnóstica foi de odontoma composto em região anterior de maxila com impactação do canino permanente. 
Figura 2 - Exame radiográfico panorâmico.

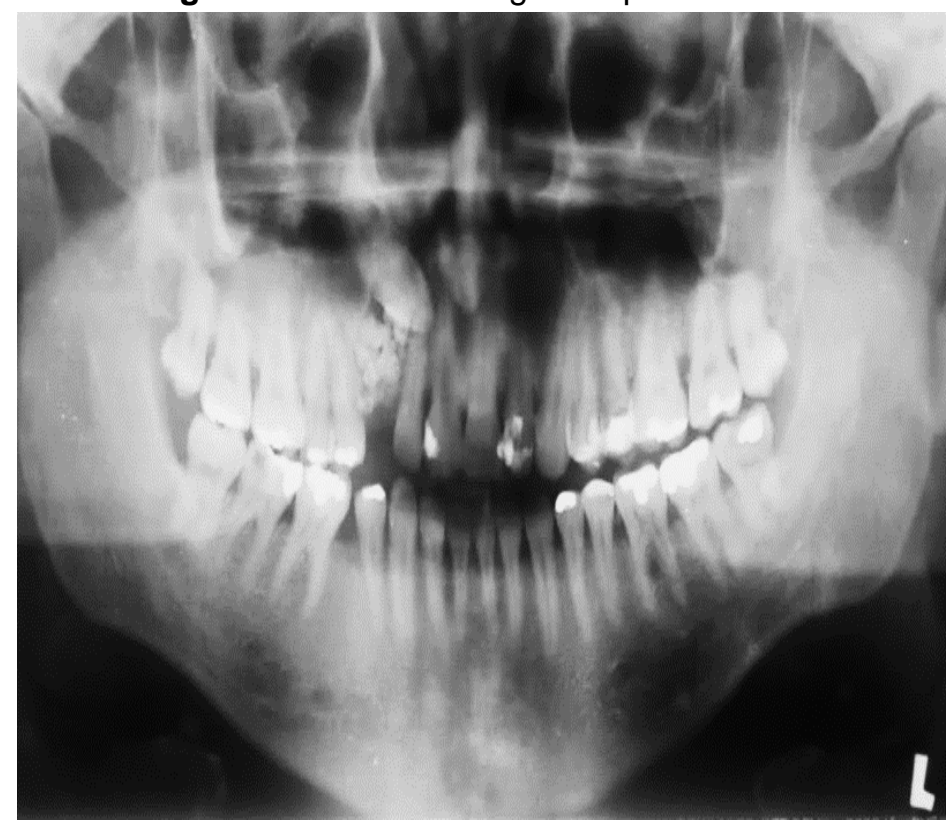

Fonte: Wanderley AEC, et al., 2019.

Diante do exposto, para confirmação diagnóstica, planejou-se a exérese da lesão em ambulatório, com exposição da lesão por meio de acesso cirúrgico, após sindesmotomia, iniciou o seccionamento da lesão em múltiplos fragmentos por meio de descoladores e curetas molt, que foram facilmente destacados do tecido ósseo adjacente., seguido de cuidadosa curetagem da cavidade afim de retirar toda a cápsula da lesão e não comprometer o elemento impactado (Figura 3).

Figura 3 - Exérese da lesão (A); Aloja Cirúrgica após remoção (B).
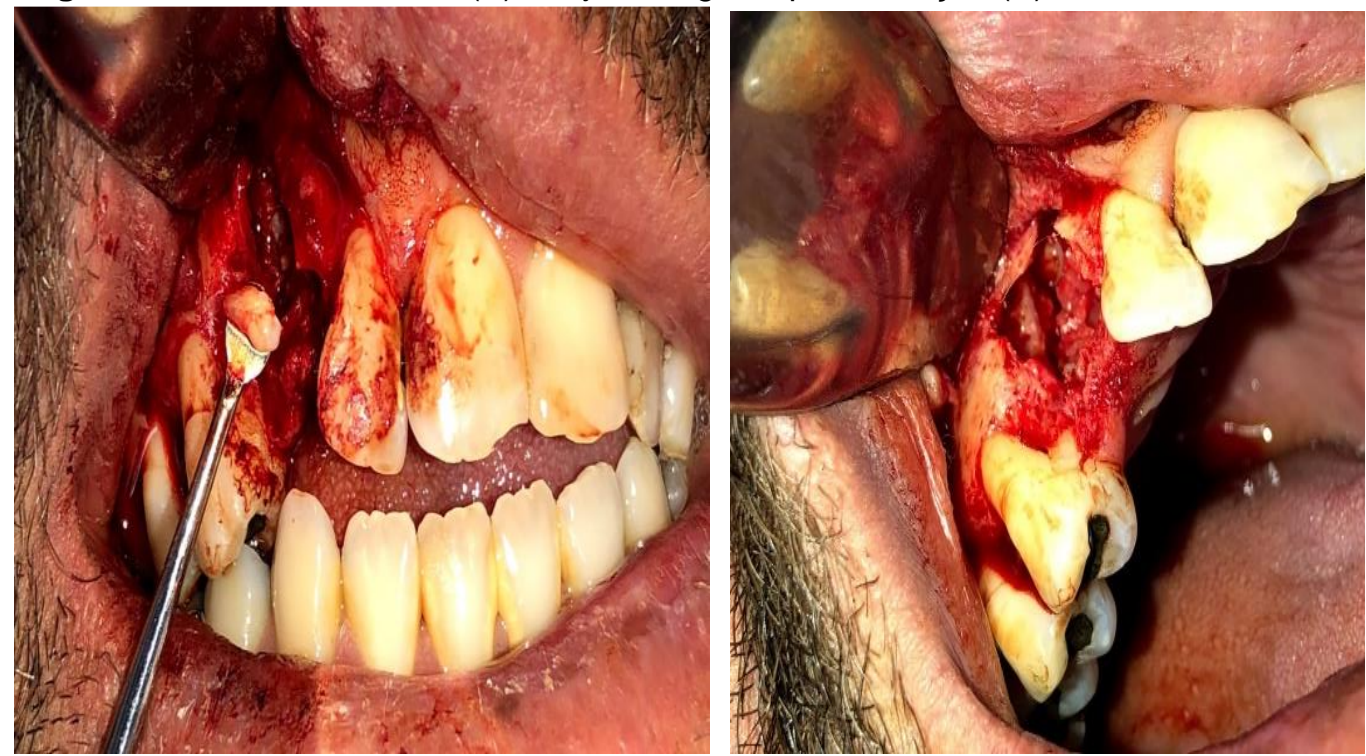

Fonte: Wanderley AEC, et al., 2019.

Parte da lesão excisada (Figura 4) foi enviada ao exame anatomopatológico, onde observou-se a presença de fragmentos de tecido calcificado semelhantes a estruturas dentárias rudimentares, formados por tecido conjuntivo denso, com somente poucas e pequenas áreas de tecido conjuntivo frouxo, confirmando assim o diagnóstico histopatológico de odontoma composto (Figura 5). 
Figura 4 - Estruturas dentárias rudimentares.

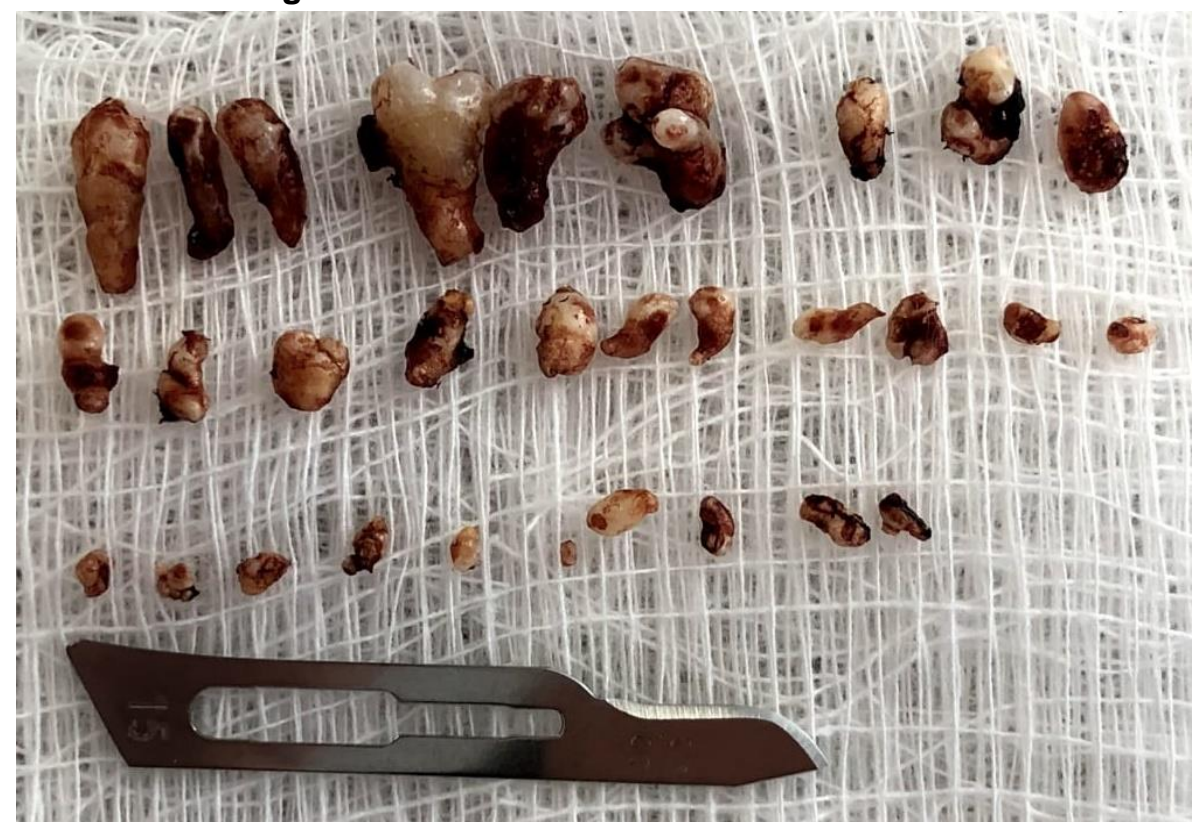

Fonte: Wanderley AEC, et al., 2019.

FIGURA 5 - Característica do Odontoma Composto em cortes histológicos

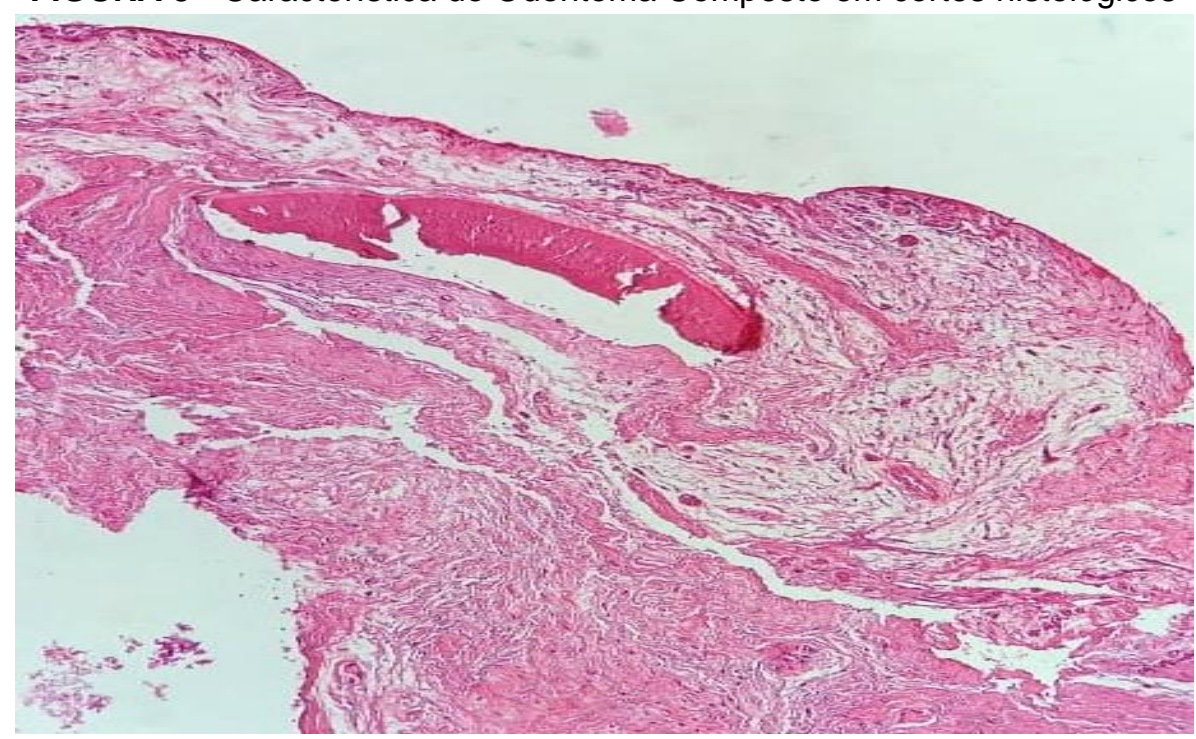

Fonte: Wanderley AEC, et al., 2019.

Em relação ao pós-operatório, o quadro progrediu sem modificações funcionais ou sensoriais, após sete dias da cirurgia, houve um excelente início da cicatrização tecidual. A nova formação de osso local será analisada no controle pós-operatório mediante radiografias panorâmicas periódicas. Paciente encontra-se em acompanhamento odontológico e aguardando planejamento ortodôntico para tracionamento do dente impactado.

\section{DISCUSSÃO}

Odontomas são lesões, em sua maioria, assintomáticos, de evolução lenta e de pequenas proporções, os quais podem passar despercebidos, gerando alterações ósseas e dentárias. Em alguns casos, a lesão pode atingir proporções maiores, levando à expansão das corticais ósseas e/ou compressão de estruturas 
nervosas, o que pode ocasionar dor (FREIRE AL, et al., 2018). No caso relatado, o odontoma causou impacção do canino permanente causando desarmonia na arcada dentária.

Pertinente a sua progressão lenta, alguns autores não aprovam as características de neoplasia verdadeira, uma vez que acontecem em métodos semelhantes aos analisados, ao longo de fases da odontogênese, isto é, em maior número de vezes, a sua evolução irrompe quando a etapa de mineralização é finalizada (PIRES WR, et al., 2013). O surgimento desta anomalia em nível molecular é destinado à alteração na via da bcatenina e ao CD44, que tem se apresentado ativamente incluído no progresso de outros tumores odontogênicos, assim como o ameloblastoma. Logo, a nível clínico, as duas patologias podem manifestar-se como elevações nodulares de consistência firme, normalmente em tecidos gengivais e em associação a dentes retidos ou não erupcionados, que se manifestou em nosso caso (CONTRERAS W, et al., 2018).

Alguns autores como Cardoso LC, et al. (2003) afirmam que odontomas compostos e complexos são diagnosticados com maior frequência no gênero feminino, divergindo assim do caso clínico em questão. Alguns relatos, sugerem não haver diferenças significativas na frequência dos odontomas relacionadas ao gênero, para Lodi CS, et al. (2014), sendo a região anterior de maxila como a mais comumente acometida, como relatou Noia CF, et al. (2008), corroborando com o caso apresentado.

Apesar de serem considerados de origem idiopática, os odontomas podem originar-se de alterações na formação dos tecidos dentários, ligadas especialmente a traumatismos e infecções (PIRES et al 2007). Entretanto, não se descarta sua origem a partir de anormalidades nos mecanismos genéticos que controlam o desenvolvimento dentário (FREIRE AL, et al., 2018). Com isso, os autores advogam não se tratar de um neoplasma verdadeiro, mas sim um tumor odontogênico com comportamento benigno (PIRES WR, et al., 2013). Inúmeros dentes têm sido correlacionados à patogênese dos odontomas. Desde o trauma no período da dentição decídua, anomalias hereditárias como síndrome de Gardner, hiperatividade dos odontoblastos ou variações nos componentes genéticos encarregados pela progressão dos elementos dentários (ESWARA UMA, 2017).

Os odontomas geralmente vêm associados a dentes não irrompidos ou discreto aumento de volume (BARNES L, et al., 2005). Em muitos casos, portanto, a lesão é descoberta durante exame radiológico como parte de um planejamento odontológico, como visto no relato do caso, em que o paciente estava ciente do dente não irrompido e aumento de volume na região do dente 13 (canino superior esquerdo) e informou durante a anamnese a presença de uma possível lesão, informação adquirida em uma consulta prévia a outro profissional durante, afirmou ainda nunca sofrido trauma no sítio de localização da patologia, divergindo do encontrado por Kämmerer PW, et al. (2016) e Pippi R (2014) que afirmam como possível causa, traumas e outras injúrias aos tecidos próximos à lesão.

Assim como o caso clínico relatado, demais estudos da literatura como os de Pires LS et al. (2007) e Cardoso LC, et al. (2003), descrevem odontomas pequenos sem sintomatologia dolorosa, que foram descobertos através de exames radiográficos rotineiros executados com a finalidade de localizar elementos dentários não erupcionados. A ausência ou falha de erupção de dentes permanentes é a manifestação clínica mais comum dos odontomas para Freire AL, et al. (2018), corroborando com os achados do relato. Clinicamente, apresentam-se como lesões pequenas, geralmente assintomáticas, podendo algumas irromper a mucosa, ocasionando uma sintomatologia dolorosa ou, dependendo da área envolvida e da expansão cortical óssea, mesmo parestesia (MATOS A, et al., 2012). O caso relatado embora não tenha manifestado nenhum grau de parestesia devido sua localização, apontou evidente expansão da cortical óssea vestibular, aumento de volume da região e discreto irrompimento de esmalte.

Para confirmação do diagnóstico final, foi proposta a realização de biópsia excisional e envio ao anatomohistopatológico, conforme ratifica Sardana D, et al. (2015) e Serra-Serra G, et al. (2009) como exame de escolha associado a tomadas radiográfica as quais confirmam a presença de regiões circunscritas, radiolucências expansivas que geralmente contêm alguns solitários ou múltiplos pequenos focos radiopacos com tamanhos irregulares que formam um produto na lesão e consequentemente a associação ao dente impactado. 
O material obtido na remoção da lesão, encontra suporte na literatura quanto ao diagnóstico estabelecido. Formado por diversos fragmentos mineralizados assemelha-se às características descritas por Matos $A$, et al. (2012). Segundo os autores, o odontoma composto é formado por um grande número de dentes rudimentares e malformados. Durante a sua formação ocorre a divisão do órgão do esmalte normal em pequenas partes, que dão forma à vários dentículos. A escolha do tratamento cirúrgico conservador concorda com as condutas adotadas em outros relatos, segundo Freire AL, et al. (2018) e Lodi CS, et al. (2014), no qual se preconiza a remoção completa da lesão com posterior encaminhamento do tecido excisado para estudo histopatológico e confirmação do diagnóstico, além do acompanhamento pós operatório com exame clínico e radiográfico para controle do caso e garantia de não recidivar. Recorrências esporádicas foram atribuídas em alguns casos relatados por Kumar LKS, et al. (2014), no entanto, estes estavam associados a remoção cirúrgica inadequada e com a lesão ainda em desenvolvimento. Alguns outros estudos demonstraram que uma enucleação conservadora é o suficiente.

A manutenção dos dentes permanentes impactados é controverso, onde alguns autores como Pontes HA, et al. (2012) afirma que não há motivo para remoção do dente envolvido, e o prognóstico é excelente com raros casos de recorrência (LOPES MLDS, et al., 2017). No entanto, enfatiza-se a rara, porém possível transformação maligna (REISER V, et al., 2013). Na maioria dos estudos, o manejo segue o adotado no referido caso, onde a manutenção deve ser viabilizada sempre que possível associado ao tracionamento e planejamento ortodôntico em casos de dentes completamente formados.

\section{CONSIDERAÇÕES FINAIS}

O odontoma composto é um tumor benigno de fácil diagnóstico clínico e por imagens, geralmente é assintomático e apesar de ter uma evolução lenta, pode atingir grandes proporções sem que o paciente perceba. Exames radiográficos de rotina ou em radiografias para investigar o motivo da não erupção de um dente, retenção de dentes decíduos ou deslocamento de dentes permanentes podem nos fornecer sinais para a descoberta do odontoma. $O$ tratamento consiste na remoção cirúrgica da lesão com base nos princípios de exodontia de dentes inclusos, sendo o prognóstico favorável.

\section{REFERÊNCIAS}

1. ABRAHAMS JM, McCLURE SA. Pediatric odontogenic tumors. Oral and Maxillofacial Surgery Clinics of North America, 2016; 28(1): 45-58.

2. AHIRE MS, et al. Odontogenic tumors: A 35-year retrospective study of 250 cases in an Indian (Maharashtra) teaching institute. Indian J Cancer, 2018, 55(3): 265-272.

3. AKERZOUL N, et al. Giant Complex Odontoma of Mandible: A Spectacular Case Report. The Open Dentistry Journal, 2017; 11 (Suppl-1, M9): 413-419.

4. BARNES L, et al. World Health Organization Classification of Tumours. Pathology and Genetics of Head and Neck Tumours. Lyon: IARC Press, 2005; 435p.

5. CARDOSO LC, et al. Odontoma combinado associado a dentes não-irrompidos: relato de casos clínicos. Rev Odontol Araçatuba. 2003; 24(2): 47-51.

6. CHRCANOVIC RB, et al. Two-stage surgical removal of large complex odontoma. Oral Maxillofac Surg. 2010; 14: 24752.

7. CONTRERAS W, et al. Peripheral Developing Odontoma or Peripheral Ameloblastic Fibro-Odontoma Erupting to Oral Cavity Case Report. Int. J. Odontostomat., 2018;12(2):117-120.

8. DE OLIVEIRA BH, et al. Compound odontoma - diagnosis and treatment: three case reports. Pediatr Dent, 2001; 23(2):151-157.

9. ESWARA UMA. Compound odontoma in anterior mandible - a case report. Malays J Med Sci, 2017; 24(3): 92.

10. FREIRE AL, et al. Odontoma composto em paciente pediátrico: relato de caso. Revista de Iniciação Científica em Odontologia, 2018; 16(3):91-99.

11. FREITAS A, et al. Radiologia odontológica. 4a ed. São Paulo: Artes Médicas; 1998; 652p.

12. GONZALEZ-ALVA $P$, et al. Podoplanin expression in odontomas: clinicopathological study and immunohistochemical analysis of 86 cases. Journal of Oral Science, $2011 ; 53(1): 67-75$. 
13. HENRIKSSON CO, KJELLMAN O. Complex odontoma. Oral Surg Oral Med Oral Pathol., 1964; 18: 64-9.

14. ISOLA G, et al. Association between odontoma and impacted teeth. Journal of Craniofacial Surgery, 2017; 28(3): 755758.

15. KÄMMERER PW, et al. Clinical parameter of odontoma with special emphasis on treatment of impacted teeth - a retrospective multicentre study and literature review. Clin Oral Investig, 2016; 20(7): 1827-1835.

16. KODALI RM, et al. An Unusual Complex Odontoma. J. Maxillofac. Oral Surg, 2010; 9(3): 314-317.

17. KUMAR LKS, et al. Ameloblastic fibro-odontoma. International Journal of Surgery Case Reports, $2014 ; 5: 1142-1144$.

18. KURAMOCHI MM, et al. Acesso extraoral para reconstrução primária em odontoma complexo raro em mandíbula.

Rev Port Estomatol Medic Dent Cir Maxilofacial, 2006; 47:35-40.

19. LODI CS, et al. Odontoma composto em paciente infantil: relato de caso. Arch. Health Invest. 2014; 3(2): $26-33$.

20. LOPES MLDS, et al. Ameloblastic fibro-odontoma: Case report and immunohistochemical profile. Journal of Oral and Maxillofacial Surgery, Medicine, and Pathology, 2017; 29: 77-82

21. MATOS A, et al. Odontoma composto em região posterior de mandíbula: relato de caso. Oral Sci., 2012; 4(2): 54-58.

22. NASCIMENTO MM, et al. Odontoma associated with impacted mandibular canine: surgically guided eruption. Rev Gaúch Odontol, 2016; 64(2): 198-202.

23. NOIA CF, et al. Odontoma composto. Rev Gaúch Odontol, 2008; 56: 213-217.

24. PIPPI R. Odontomas and supernumerary teeth: is there a common origin?. Int J Med Sci, 2014; 11(12): $1282-1297$.

25. PIRES LS et al. Odontoma: estado da arte e relato de caso clínico. Stomatos, 2007; 13: 21-29.

26. PIRES WR, et al. Odontoma complexo de grande proporção em ramo mandibular: relato de caso. Rev Odontol UNESP, 2013; 42(2): 138-143.

27. PONTES HA, et al. Report offour cases of ameloblasticfibro-odontoma inmandible and discussion of the literature about the treatment. J Craniomaxillofac Surg, 2012; 40: 59-63.

28. PREOTEASA CT, PREOTEASA E. Compound odontoma - morphology, clinical findings and treatment. Case report. Rom J Morphol Embryol, 2018; 59(3): 997-1000.

29. REISER V, et al. Pediatric ameloblastic fibro-odontosarcoma of the mandible: a challenge of diagnosis and treatment. J Oral Maxillofac Surg, 2013; 71: e45-e57.

30. SANTOS JN, et al. Odontogenic tumors: analysis of 127 cases. Pesqui Odontol Bras, 2001; 15(4): 308-313.

31. SARDANA D, et al. Surgical excision of large ameloblastic fibro-odontoma followed byalloplastic bone graft-2 year follow-up: A case report. Journal of Oral and Maxillofacial Surgery, Medicine, and Pathology, 2015; 27: 41-44.

32. SATISH V, et al. Odontome: a brief overview. Int J Clin Pediatr Dent, 2011; 4(3): 177-185.

33. SERRA-SERRA G, et al. Erupted odontomas: a report of three cases and review of the literature. Med Oral Patol Oral Cir Bucal, 2009; 14(6): 299-303. 